\title{
EL IMPACTO DE LA COMUNICACIÓN PÚBLICA EN EL DESARROLLO DEL TURISMO INTERNO EN EL ECUADOR
}

\author{
THE IMPACT OF PUBLLC COMMUNICATION IN THE DEVELOPMENT OF \\ DOMESTIC TOURISM IN ECUADOR
}

\section{GABRIELA ESMERALDA GRACIA REYES' ${ }^{1}$, PATRICIA DOLORES TORRES FUENTES}

\author{
Universidad Católica de Santiago de Guayaquil. gabriela.gracia@cu.ucsg.edu.ec \\ 2 Universidad Católica de Santiago de Guayaquil. patricia.torres@cu.ucsg.edu.ec
}

El turismo y su importancia en el marco de la economía ecuatoriana toma cada vez más fuerza y se convierte en una industria muy prometedora. De ahí nace la necesidad de extender discusiones sobre la comunicación y turismo, articulados mediante las políticas públicas del actual gobierno como medio y mediador entre los diferentes actores estratégicos. Este trabajo pretende despertar el interés de los lectores sobre el impacto y difusión de la comunicación pública en las agendas de promoción turística nacional; y tiene como objetivo identificar cómo el diario público de mayor circulación nacional, El Telégrafo, promueve la circulación de temas turístico. Se utilizó la metodología de análisis de contenido en 365 publicaciones del diario, en el marco de una interrelación en la que medios masivos, el Estado y las unidades estratégicas del sector aparecen como los actores de la divulgación, en un sistema que -para mantenerse- exige la directa participación de cada uno de sus integrantes. Adicional, se midió la intencionalidad de la comunicación pública y el nivel de éxito de la estrategia de Gobierno en promover el desarrollo del turismo interno. Se llegó a la conclusión de que el diario El Telégrafo da mayor cobertura a los organismos gubernamentales en ditrimento de los colectivos sociales.

PALABRAS CLAVE: comunicación pública, turismo interno, Ecuador, medios públicos, análisis de contenido.
Tourism and its importance in the context of the Ecuadorian economy increasingly takes more strength and becomes a very promising industry. This gives rise to the need to extend discussions on communication and tourism, articulated through public policy of the current government as a means and mediator between different strategic actors. This work aims to arouse the interest of readers about the impact and dissemination of public communication on the agendas of national tourism promotion and aims to identify how the public daily national circulation, El Telegrafo, promotes the circulation of tourist topics. The methodology was used for content analysis of 365 publications of the journal, in the framework of an interrelation in which massive media, the State and units of the strategic sector appear as the actors of the disclosure, in a system that requires to keep the direct participation of each of its members. Additionally, the intentionality of public communication and the level of success of the government strategy to promote the development of domestic tourismwas measured. It was concluded that the journal El Telegrafo gives more coverage to the government agencies in ditrimento of social collectives.

KEYWORDS: public communication, domestic tourism, Ecuador, public media content analysis. 


\section{INTRODUCCIÓN}

Muñoz Mazón, Fuentes Moraleda, y Fayos-Solà en su artículo Turismo como instrumento de desarrollo: Una visión alternativa desde factores humanos, sociales $e$ institucionales sostienen que:

"El auge del turismo en algunos lugares ha coincidido con el avance de su economía, por lo que en ocasiones se ha podido confundir el crecimiento turístico con el desarrollo que éste podría producir en las sociedades. [...] Aunque el turismo es capaz de crear empleo, generar ingresos e, incluso, fomentar infraestructuras, ello no significa que sea un factor de desarrollo en todos los casos. $\mathrm{Ni}$ siquiera que tenga necesariamente connotaciones de programa pro-pobre. Lo que determina que el turismo sea un instrumento para el desarrollo son determinadas políticas y apropiadas reglas de juego. En ausencia de ellas, el turismo puede igualmente devenir en una actividad anti-pobre" (Muñoz Mazón et al., 2012: 444).

Otro de los beneficios del turismo para los propios ecuatorianos es la capacidad de contribuir a la transmisión de saberes ancestrales, culturales e históricos y fomentar la identidad nacional en colectivos sociales dispersos e inequitativos. Es destacable la importancia de la cooperación público-privada y la gobernanza si se quiere utilizar al turismo como alternativa óptima de reducción de la pobreza y mejora de la calidad de vida, lo señalan los profesores de la Universidad La Rioja de España, Muñoz Mazón et al. (2012).

Otro conjunto de consideraciones que hacen referencia Muñoz Mazón et al. es sobre los enfoques teóricos versus la práctica. Consideran al turismo como un instrumento de desarrollo que debe consistir en un agregado de programas y acciones coordinados, y señalan que:

"Podemos encontrar consenso al aseverar que esta coordinación y la eficiencia total del agregado dependerán de que los programas y acciones sean congruentes con metodologías bien establecidas, prácticas de probado éxito, etc. Las metodologías para la política turística y su gobernanza comprenden desde herramientas analíticas tales como las Cuentas Satélite del Turismo y la inteligencia de mercado a planes de política turística con programas dedicados a la sostenibilidad, gestión del conocimiento, innovación, promoción, calidad y excelencia. [...] El papel de las instituciones, globales y locales, es reso- lutorio en esta búsqueda" (Muñoz Mazón et al., 2012: 445 - 446).

Como se sustenta ampliamente el turismo constituye una función económica a considerar y desfila activamente en el Plan Nacional para el Buen Vivir y la Matriz Productiva del gobierno del Ecuador. Actualmente esta industria se ubica en el tercer lugar de las aportaciones a la economía ecuatoriana según la Coordinación Ceneral de Estadísticas e Investigación del Ministerio de Turismo del Ecuador (2015), y adquiere mayor interés en el acontecer noticioso dada su materia de sostenibilidad y potencialidad para contribuir a la salida de la crisis económica (Ponce, 2011).

Es oportuno por tanto, según Ponce (2011), que si se apuesta por fomentar este sector se debe obtener diagnósticos que ayuden en los procesos de toma de decisiones y actuaciones en este campo.

\section{DE LA COMUNICACIÓN Y EL TURISMO A LA COMUNICACIÓN PÚBLICA}

Autores como Crompton, Fakeye, Govers y Co, Hankinson, O'Leary y Pike (citado en Ortega y Rodríguez, 2007) declaran firmemente la importancia de la comunicación para la promoción de los destinos turísticos, ya que permite estimular el interés de los turistas para que los conozcan y recuerden su existencia y atractivo. De esta forma se habla de una comunicación que legitimice el poder de la ciudadanía, entendiéndola en sus diferentes dimensiones sociales, culturales y económicas, y desamarre el interés particular de unos cuantos en los medios de comunicación pública.

Así, Ortega y Rodríguez (2007: 2) cita a destacados autores (McKercher, et al.) relacionados a continuación que manifiestan que una correcta comunicación de los recursos y productos turísticos en los destinos se traduce en comportamientos y actitudes como las siguientes:

- Presencia de los turistas en un mayor número de lugares o atracciones que la inicialmente prevista (McKercher, 2001).

- Ampliación de la estancia para conocer lugares o atracciones sobre los que los turistas no tenían un conocimiento previo (Fesenmaier, Vogt y Stewart, 1993; Vogt y Andereck, 2003).

- Modificación de la imagen del destino derivada de un mayor conocimiento de la oferta turística existente y de las ex- 
periencias vividas con la misma (Vogt y Andereck, 2003).

- Actitud más favorable para regresar al propio destino elegido para visitar los nuevos lugares de los que se ha tenido conocimiento y que no han podido ser visitados (Vogt y Andereck, 2003).

- Mayor conocimiento de la oferta turística local y regional para trasladar a familiares y amigos, lo que ejerce una fuerte influencia en la elección de los destinos de estas personas sin incurrir en costes de comunicación alguno (Fodness y Murray, 1999; Pechlaner y Raich, 2002; Money y Crotts, 2003).

Según Beauchamp (citado por Demers y Lavigne, 2007: 67) define a la comunicación pública como:

"El conjunto de fenómenos de producción, tratamiento, difusión y retroacción de la información que refleja, crea y orienta los debates y los temas públicos; la comunicación pública entendida no solamente como el quehacer de los medios, sino también de las instituciones, las empresas, los movimientos y los grupos que intervienen en la plaza pública” (Beauchamp, 1991: XIII).

Demers y Lavigne sostienen que:

"La comunicación pública apunta al debate público y se preocupa, por las condiciones de su existencia y por los mecanismos sociales de su surgimiento [...] no se interesa por todo a lo que se le da visibilidad en la plaza pública, sino, esencialmente, por aquello que va a contribuir en la formación de la opinión pública y en las eventuales decisiones y acciones gubernamentales y estatales" (Demers y Lavigne, 2007: 72-73).

Es aquí donde los observadores críticos ven a los medios como:

"Instrumentos del comercio de masas y como empresas de educación para el consumo. [...] Este enfoque sobre los medios de comunicación se extiende bajo el concepto de comunicación pública, desde las prácticas de las campañas de prensa (periodismo), de relaciones de prensa y de publicidad (ofertas, anuncios), hasta el de las prácticas de "intervención en comunicación social", donde "intervención" designa las actividades mediáticas para cambiar las opiniones, las actitudes y los comportamientos, y "comunicación social” designa las intervenciones comunicacionales que apoyan las causas consi- deradas como socialmente buenas" (Demers y Lavigne, 2007: 75).

De esta forma, afirman Demers y Lavigne (2007: 77), "La comunicación pública hace de los medios uno de los mecanismos a través de los cuales los actores sociales se hablan entre sí, siendo el Estado sólo un actor más".

Para López (2002), Demmers y Lavigne (2007) el análisis de los contenidos mediáticos permite deducir la intención de comunicación, medir la motivación y aspectos relevantes como: el fondo y forma en que son abordados los temas de interés del público seleccionado, el ángulo y la sensibilidad del producto y la participación de la ciudadanía en los medios mismos.

\section{DESARROLLO}

La comunicación en y para los destinos turísticos puede realizarse por diversos medios, entre los que podemos citar a los medios personales e impersonales y de estos últimos desagregarlos en los espacios de la televisión local, los diarios locales, la prensa gratuita, los folletos, las guías de atracciones y espectáculos, los libros turísticos, los vídeos o películas, el teletexto e Internet (Ortega y Rodríguez, 2007).

En el estudio se tomó como unidad de análisis el Diario El Telégrafo, fundado el 16 de febrero de 1884 en la ciudad de Guayaquil y de circulación nacional. Desde su fundación, este medio, conocido como el Decano de la Prensa Nacional, ha mantenido una organización de carácter familiar. Tras estar en manos privadas por más de 100 años, el diario fue incautado y pasó al Estado ecuatoriano en el 2008, que es propietario de la mayoría de sus acciones por medio del Ministerio de Telecomunicaciones y desde esa fecha dirige la línea editorial del medio, sin detener su circulación. Se ha considerado para el periodo de análisis los meses de abril de 2014 a marzo de 2015, lo que definió el tamaño de la población de 365 días.

Como metodología se escogió el análisis de contenido como modo de identificar y examinar los mensajes en donde se expliciten temas referentes a turismo interno. Según Lalande (citado en López, 2002) se puede considerar al análisis como la descomposición de un todo en sus partes. Mientras que a juicio de Bardin (1986) es:

"un conjunto de instrumentos metodológicos, aplicados a lo que él denomina como "discursos" (contenidos y continentes) extremadamente diversificados. El factor común de estas técnicas múltiples y multiplicadas -desde el cálculo de frecuencias suministradoras de 
datos cifrados hasta la extracción de estructuras que se traducen en modelos- es una hermenéutica controlada, basada en la deducción: "la inferencia»" (citado en López, 2002: 173 - 175).

Del modo más simple y general, se puede decir que el análisis de contenido es un tipo de medición aplicado a un mensaje, en el marco de propósitos del ámbito de las ciencias sociales. Es evidente que representa una de las técnicas más elaboradas y la que goza de mayor prestigio científico en el campo de la observación documental (López, 2002).

Los aspectos que se consideraron dentro del análisis de contenido fueron: día y fecha de publicación, título de la nota, sección dónde se publica, número de página (ubicación dentro del diario), resumen explicativo de la noticia, porcentaje de publicidad del diario por día, porcentaje de contenido del diario por día, fuentes emisoras de noticias y precio.Comportamiento de la presencia de notas turísticas.

Siguiendo la literatura expuesta, se determinó que diario El Telégrafo tiene un ratio de publicación de 16 notas por mes de temas relacionados al turismo, notas que están dentro del género periodístico noticia y son parte de la coyuntura social o interés público. Obsérvese la Figura 1 a continuación.

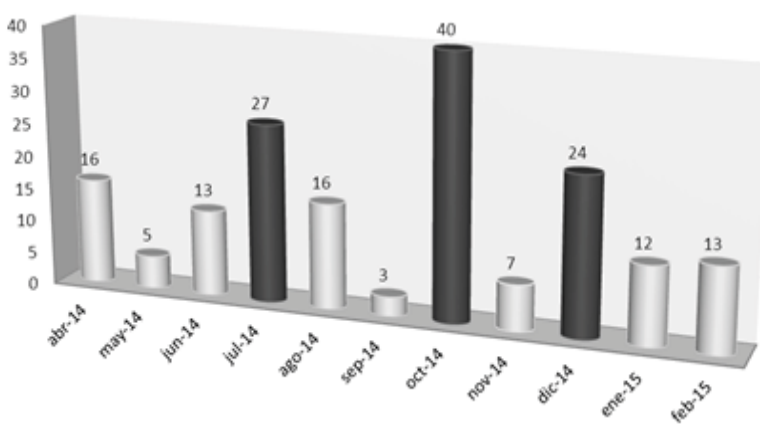

Figura 1. Número de noticias por mes Fuente: Diario El Telégrafo

De los 365 ejemplares analizados, el 33\% sí contaba con al menos una noticia de turismo; siendo septiembre el mes con menor cantidad de ejemplares (3) y número de noticias (3) y el mes de octubre el opuesto con 20 ejemplares con 40 noticias. Este comportamiento se refleja en la Figura 2.

El diario El Telégrafo tiene un promedio de 63 páginas diarias siendo los días lunes y domingo en donde sus ejemplares han llegado hasta un promedio de 75 y 105 páginas respectivamente.

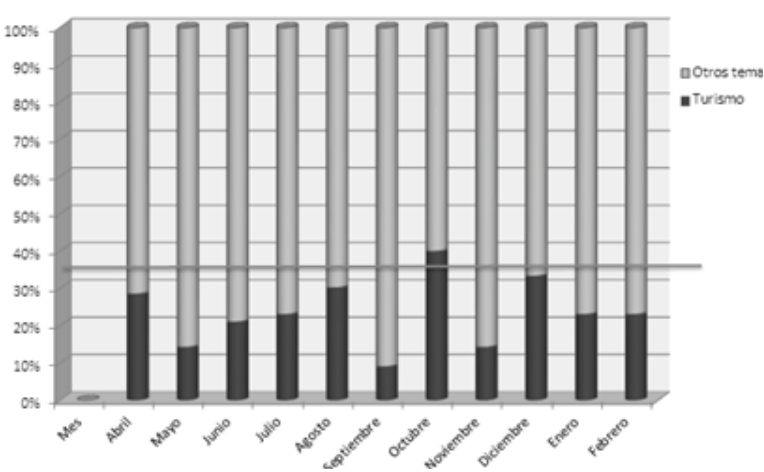

Figura 2. Cantidad de noticias por ejemplar Fuente: Diario El Telégrafo

Al analizar en qué secciones se publican estas noticias se descubrió que Ciudadanía, Actualidad y Sociedad son las que con mayor recurrencia dan espacios a los contenidos de turismo y recogen lo que distribuyen las fuentes públicas como Ministerios, Alcaldías y otros organismos estatales. Se pudo apreciar que este tipo de publicaciones no se distribuyen por todo el diario sino en el bloque Página Dos y bloque $B$ tal y como puede observarse gráficamente en la figura 3. En el bloque $C$ no pudo constatarse presencia de notas turísticas.

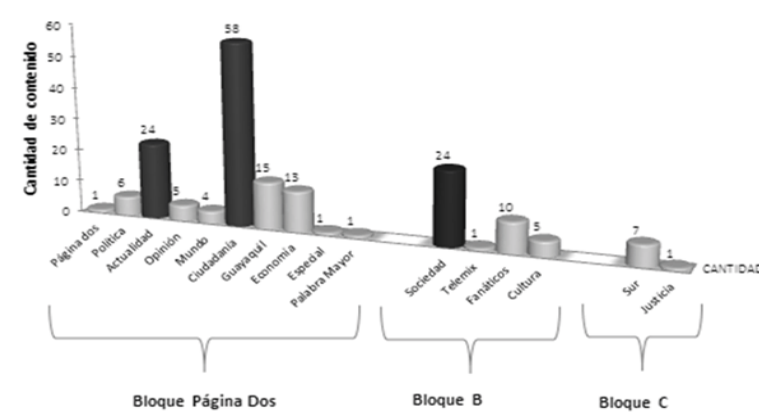

Figura 3. Secciones del diario donde se han publicado noticias de turismo Fuente: Diario El Telégrafo

Otro dato a resaltar es que de los meses analizados no existe un promedio similar de publicaciones de noticias de turismo. Hay meses en que las publicaciones sobre estos temas son mínimas y se privilegian otro tipo de noticias coyunturales, por ejemplo las políticas o económicas, o por la estacionalidad del sector amparadas en las vacaciones de la región costa o sierra del país se ve mayor recurrencia de cobertura noticiosa de esta índole.

CONCLUSIONES

La comunicación de los destinos turísticos debe ser contemplada como una actuación estratégica, para que a través de ella se pueda dar a 
conocer la existencia de lugares, atracciones y recursos turísticos existentes en los ámbitos locales y regionales del Ecuador. Como va siendo obvio, no es propósito de estas páginas analizar con detenimiento las políticas de gobierno, ni la agenda setting del medio de comunicación. Lo que interesa subrayar, más que todo, es el reconocimiento creciente que las instancias de poder han encontrado como fuente del consenso social a través de la comunicación pública.

Aunque esta investigación ha centrado su estudio en la prensa como generadora de cambios en el turismo interno, es obvio que el desarrollo de la comunicación pública contemporánea no es la única que aporta para lograr el mismo propósito.

El hecho de que el desarrollo del sector del turismo dependa en gran medida del grado de visibilidad alcanzado en los medios, confirma la centralidad de la comunicación en los procesos de formación de una sociedad con conocimiento. El presente estudio ha intentado sortear varios obstáculos en la investigación sobre las perspectivas multidisciplinarias, entre ellas la necesidad de abordar el análisis de contenido desde su naturaleza política y comunicativa.

De cara al futuro, sin embargo, la investigación en este campo tendrá que afrontar todavía varios desafíos, derivados fundamentalmente de la necesidad de vincular los diferentes actores (ciudadanía, empresas del sector del turismo, organismos estatales, gremios y asociaciones, etc.) que intervienen en el proceso de formación de opiniones e integrar múltiples aportaciones.

La información obtenida revela una heterogeneidad en cuanto a los espacios que le otorga el diario público El Telégrafo, en tanto el tema sea coyuntural o la temporalidad - vacaciones - lo amerite. Esto profundiza aún más la estacionalidad del sector y no ayudan a generar mayores flujos constantes de turismo a lo largo del año.

Se puede afirmar que en la actualidad el medio público no se identifica con el colectivo empresarial del sector del turismo sino mayormente con los organismos estatales quienes objetivizan la difusión de los destinos dejando en la ambigüedad a los demás actores estratégicos del sector tales como: hoteles, transportes turísticos, agencias de viajes operadoras, centros de entretenimiento, etc.

El producto turístico de interior del Ecuador dispone de un recurso de gran potencial, un colectivo social de emprendedores, un tejido de micros y pequeña empresas que se mues- tra activo y absolutamente receptivo ante el aprendizaje de todo cuanto posibilite éxito en el desempeño de su actividad.

El turismo interno, a pesar de la evolución positiva en los últimos años, no ha activado suficientemente las sinergias locales ni la transversalidad que se le atribuye y le es propia, un hecho que lo constata es que los medios públicos siguen teniendo como principal fuente a los organismos estatales y no a la ciudadanía. Conviene insistir en la necesidad de seguir realizando diagnósticos del sector y del territorio, para considerarlos en políticas de desarrollo.

\section{REFERENCIAS BIBLIOGRÁFICAS}

Coordinación General de Estadística e Investigación del Ministerio de Turismo del Ecuador, Marzo (2015). Principales indicadores de turismo. Recuperado de http://servicios.turismo.gob.ec/descargas/Turismocifras/BoletinesEstadisticos/TurismoReceptor/ Boletines-estadisticos-reporte-mensual-de-turismoreceptor-marzo-2015.pdf

Demers, F. \& Lavigne, A. (2007). La comunicación pública: una prioridad contemporánea de investigación. Comunicación y Sociedad, (8) 65-87. Recuperado de http://www.redalyc.org/articulo.oa?id=34600804.

Diario El Telégrafo. (2015). Ediciones impresas (2). Recuperado de http://www.telegrafo.com.ec/edicionesimpresas/el-telegrafo.html

López Noguero, F. (2002). El análisis de contenido como método de investigación. XXI, Revista de Educación, (4)167-179.

Muñoz Mazón, A. I., Fuentes Moraleda, L. \& Fayos-Solà , E. (2012). Turismo como instrumento de desarrollo: Una visión alternativa desde factores humanos, sociales e institucionales. PASOS. Revista de Turismo y Patrimonio Cultural, 10(5) 437-449.

Ortega Martínez, E. y Rodríguez Herráez, B. (2007). La Comunicación en los Destinos Turísticos: Percepción de los Residentes en España. Ponencia presentada en elXIXCongreso anualy XV Congreso Hispano Francés de AEDEM, Madrid, España. Recuperado de http:// dialnet.unirioja.es/descarga/articulo/2482215.pdf

Ponce Sánchez, M. D. (2011). Competitividad e Innovación en el Producto Turístico Rural de la Región de Murcia. Cuadernos de Turismo, (27) 743-758.

Secretaria Nacional de Planificación y Desarrollo (Senplades). (2013). Plan Nacional para el Buen Vivir 2013 - 2017 (1era ed.). Quito, Ecuador.

Secretaria Nacional de Planificación y Desarrollo (Senplades). (2012). Transformación de la Matriz Productiva. Revolución Productiva a través del conocimiento y talento humano (1era ed.). Boletín Informativo. Quito, Ecuador. 